\title{
F-Formations in Cooking Together: A Digital Ethnography Using YouTube
}

\author{
Jeni Paay ${ }^{1}$, Jesper Kjeldskov ${ }^{1}$, Mikael B. Skov ${ }^{1}$, and Kenton O’Hara ${ }^{2}$ \\ ${ }^{1}$ Aalborg University, 9220 Aalborg, Denmark \\ ${ }^{2}$ Microsoft Research Cambridge CB3 OFB, UK \\ \{jeni,jesper, dubois\}@Cs.aau.dk, kenton@gwork.org
}

\begin{abstract}
Cooking together is an important part of our lives. We cook with others not only to create a meal, but also to enhance our relationships. But how does this role of communal cooking translate into modern society where families and friends are increasingly separated physically and connected primarily online? Motivated by this question we have embraced research into the design of future networked cooking spaces. The first step has been to understand how people use physical space while cooking together. Through a digital ethnography on YouTube videos, we have analyzed the spatial configurations of people, food and technology based on Kendon's notions of spacing and orientation. Our main contribution is the identification of known F-formations as well as new formations taking place during social cooking. Based on this we suggest that given the presence of formations in the kitchen different from those found during activities that are mainly conversational, simply installing traditional video-conferencing systems in people's kitchens will not suffice in facilitating the interactions taking place there. Instead, designers need to rethink the positioning and use of cameras and displays.
\end{abstract}

Keywords: Cooking, F-formation, proxemics, digital ethnography, YouTube.

\section{Introduction}

Recently, the kitchen has become a focus for HCI research. Cooking together is an important part of our lives. We cook with friends, families, colleagues and strangers to share not only the experience of creating a meal, but in the spirit of commensality, as a social event where we enhance our relationships with others through shared stories, relating daily happenings and discovering new ideas about food preparation from each other. Given this role of food and the kitchen as a place for social interaction, it is important to understand the role computer technology might play in this context now and in the future [8]. But how does this role of communal cooking translate into modern society where families and friends are increasingly separated physically and connected primarily online?

Motivated by this question, we have embraced research into on future cooking spaces that enable people to have meaningful and positive social food experiences 
while cooking together, but in different places. As with environments for the work domain [29], future kitchen environments may integrate designs that provide geographically distributed people with the experience of cooking with remote family and friends using their respective kitchens as one shared digitally "blended" cooking space. This, we believe, would give people the opportunity to interact socially with close ones living in another place as an integrated part of their daily lives and household routines, which are important aspects of keeping distant relatives and friends connected [26]. Fundamental to this, we think it is important that people sharing a blended cooking space experience the same essential qualities that make collocated human-food interaction meaningful.

Subscribing, as we do, to the view that kitchens are "sites where meaning is produced, as well as meals" [1] has some profound consequences for the way one does HCI research in this domain. Firstly, it becomes more about people and their interactions in and around the kitchen, rather than just about building new technology to assist kitchen tasks. Secondly, if we wish to design compelling and meaningful new technologies that might enrich these interactions, we need to study people's practices in the kitchen and unravel and understand the richness of social interactions and experiences that happen there while cooking together.

In this paper, we explore how people arrange themselves spatially while cooking together with a "remote viewer" sharing the experience, and how new methodologies may be devised for studying this empirically. Specifically, we seek to better understand the activity of shared cooking: people's interactions with co-present others, with the physical kitchen layout, with cooking artifacts, and with remote viewers watching on video. As a source of data for this study we have turned to the vast amount of usergenerated digital video material available on YouTube depicting people cooking together in private kitchens and wanting to share this social experience with an outside audience through the Internet. Analyzing this video material can be viewed as a form of "digital ethnography" [22] where the phenomena of interest are not observed firsthand but are mediated through digital data. This approach, we believe, has great potential for informing interaction design by giving us insight into human behavior and activity in real world settings, such as private and domestic life, that are inherently difficult to reach with traditional ethnographic techniques.

The paper is structured as follows. First we provide a background of related work in two areas: HCI in the kitchen, and analysis of human spatial configurations. We then turn our attention to the specific digital ethnography of cooking together that we have carried out, describing its methodological motivations and challenges and the details of the observational and analytical procedures followed. This is followed by a detailed account of our findings, describing and illustrating the formations identified in the videos, and the activities observed for creating and sustaining them. In the discussion section we take a step back from our empirical findings and reflect on their significance and implications. As the methodology used in our study is also of some novelty to the $\mathrm{CHI}$ community we also reflect on this approach to studying phenomena in domestic settings for the purpose of interaction design. Finally, we conclude on our work and outline plans for further work towards informing design. 


\section{Background and Related Work}

Our research draws on two areas of related work: 1) HCI in the kitchen, and 2) theories about how people organize themselves in physical space.

\subsection{HCI in the Kitchen}

A number of research efforts have begun exploring digital technologies in the support of cooking. These have included Cooks Collage [35], CookingNavi [10], Cooking with the Elements [4], The Living Cookbook [34], Not Enough Cooks in the Kitchen [33], CounterActive [17], Kitchen Album [20], and PersonalChef [23]. These technologies vary in terms of how they approach cooking and requirements. For some, the approach has been to support the functional aspects of cooking and overcoming various difficulties with it (e.g. [35] and [10]). For others attention has been on the social meanings and practices of cooking.

According to Grimes and Harper [8], research on HCI in the context of kitchens has for the most part neglected technology design that focuses on the social experiences that we have with each other in the context of cooking and eating. Instead, the overwhelming trend has been to design "corrective technology" applications that act as a remedy for perceived problems that people have with planning, preparing and consuming food [8]. As an alternative, Grimes and Harper advocate a focus on what they call "celebratory technology" - technology that gives us positive, delightful, pleasurable and exciting experiences with food - as a potential new research and design space in HCI. Likewise, in their kitchen manifesto, Bell and Kaye [1] advocate a kitchen/technology relationship that draws on and learns from the rich cultural history of the kitchen as a place for living and above all, a focus on those who experience the space, rather than the resident technologies. The manifesto calls for a focus on experience over efficiency and an understanding of the use of objects in the kitchen context.

Exemplifying this line of thought, research on HCI in the kitchen has recently and increasingly turned its attention to the social meanings and practices of cooking in which the kitchens are viewed as "sites where meaning is produced, as well as meals" [1]. For example, sharing recipes as a social act (e.g. [34]) or providing mentoring support for friends and family while they learn to cook (e.g. [33]).

In The Living Cookbook [34], people's cooking experiences are recorded and shared in an interactive digital medium containing video and annotated audio that others may play back later. The agenda is to enable people to share cooking experiences, to educate others in cooking practice, and to suggest a sense of presence and sociability in the cooking activity. The role of technology in this design is to support intimacy, communication, education, fun and creativity in the cooking experience. It also allows people to strengthen cultural and social connections with others, and to stimulate cross-cultural as well as cross-generational interaction. 
In Not Enough Cooks in the Kitchen [33], video conferencing and video archiving are used to facilitate collaborative cooking experiences, and to receive, record and keep cooking tips from remote friends and family. The main purpose is the teaching and learning of cooking skills by providing a medium for experienced cooks to share their knowledge with novices and giving them valuable life skills and digital keepsakes of the experience. Designed to replace phone calls and emails between family and friends when trying to learn how to cook, it caters for much more of the richness of collocated cooking, and makes cooking with remote partners a socially enjoyable experience.

Similar to these examples, our focus here is concerned with the shared experience of cooking, and using technology for bringing people across different households together socially in shared spaces for cooking. But in order to meaningfully inform technology design that are both useful and sensitive to the social and physical context that they are to operate in, we need to understand better people's daily practices in these spaces [1].

\subsection{Proxemics and F-Formations}

As discussed by Rogers [32], a number of theories from other disciplines, new or relatively unknown to the field of HCI, are currently making their way into our field. Some of these, she argues, represent "the beginnings of a "turn to space", including schematic frameworks for modeling people's interactions with each other in physical space, such as proxemics and F-formations. Developed for analyzing social interactions outside the technology domain, these theories and frameworks have recently proved to be highly valuable in relation to interaction design as this domain broadens itself out to embrace the challenges of digital ecologies and pervasive and ubiquitous computing environments.

Proxemics covers a topic concerned with peoples' use of their immediate physical space in their interaction and communication with each other. It was originally developed by Edward Hall, a cultural anthropologist, in the early 1960s [9], but has recently gained renewed momentum in HCI as a framework for also describing and understanding people's interaction with devices and displays around them. In brief, Hall proposed that (in the USA) four interpersonal distances govern social interaction: intimate $(0-0.46 \mathrm{~m})$, personal $(0.46-1.22 \mathrm{~m})$, social $(1.22-3.66 \mathrm{~m})$ and public $(>3.66 \mathrm{~m})$. In the context of HCI this provides a useful framework for analyzing and designing interactive technology. Examples of this include Obata and Sasaki' virtual visiting system [28] and Heaton's case studies of social interaction in virtual workspaces [13] in a CSCW context, and more recently in a more general interaction design context, the works of Greenberg and colleagues on Proxemic Interactions [7].

F-formations is a part of a conceptual framework about people's interpersonal spacing and orientation developed by Adam Kendon, a leading researcher in the study of gesture, in the late 1970s [18] [19]. The notion of F-formations helps explain how people arrange themselves spatially with respect to others, and to artifacts in different kinds of social interactions and collaborations and can also be used to explore the influence of physical environment on the interpersonal interactions taking place there. 
It was developed by Kendon in response to previous studies in proxemics in order to draw attention to the fact that "participants in occasions of interactions of all sorts may enter together into a cooperative relationship to sustain, through time, a more or less constant pattern of spatial orientational arrangement" [Kendon 2012, personal communication]. Briefly described, in an F-formation system individuals have a spaced called a transactional segment, which is the space where they focus attention and manipulate artifacts. This space is defined in relationship to their lower body, and changes in size depending on the kind of activity people are doing. An F-formation is formed when the transactional segments of two or more people overlap and create a shared inner space, where the shared activity of those people occurs. This is called the o-space [18] or the use-space [19]. In the latter Kendon gives a series of concrete examples of different types of F-formations in interpersonal communication, such as the L-shaped formation of people standing orthogonal to each other, the vis-à-vis formation of facing each other directly, the side-by-side formations of facing the same direction, and the semi-circular formation of more than two people side-by side curving inwards to speak to each other.

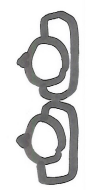

(a)

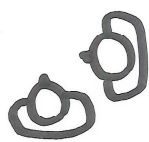

(b)

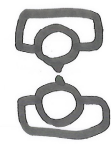

(c)

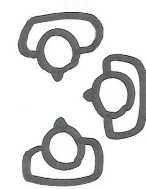

(d)

Fig. 1. Schematic examples of different F-formations:

(a) side-by-side, (b) L-shaped, (c) vis-á-vis, (d) semi-circular

Like proxemics, the work on F-formations has recently attracted interest among HCI researchers concerned with design and use of technology embedded in the physical environment, for example in [14] [15] [21] [24] [25] [29]. Marshall et al. [21] use the F-formation system for analyzing social interactions between visitors and staff in an information center for tourists. They show how the presence or absence of F-formations can explain the relationship between co-located interactions and the physical environment, and how this may inform technology design. Mentis et al. [24] conduct a similar analysis but in the context of work practices in neurosurgery theatres. This leads to insight about the spatial organization of shared work practices in this specific setting, and to understanding design implications for touchless interaction with medical technology. Other uses of F-formations in HCI include investigating the spatial relationships between humans and service robots during cooperative tasks [15], and detecting F-formations from video footage for analysing social interactive behaviour in crowded environments [14].

Spatial Organization in the Kitchen. Our concerns with the spatial organisation of behavior in the context of shared cooking lies in the fact that architectural design of kitchen spaces, like other spaces [21], structure the kinds of interactions and social activities that are enacted within them. People also adapt their activity patterns in respect to artifacts and others they are interacting with, and the very activity of 
cooking influences the ways in which human interaction is spatially organized in the kitchen. Working side by side at a kitchen bench, for example, influences how people communicate as opposed to working at opposite sides of a kitchen island. Facing a video camera during the interaction adds yet another level of complexity, not only to local communication, but also to the viewers' perceived distance from the cook, which affects the intimacy of the interaction. Issues such as eye gaze and field of view can all affect the nature of the interaction. How these physical aspects contribute to shaping the experience people have when cooking together is what we have aimed to study and understand.

\section{A Digital Ethnography of Cooking Together}

Our focus in this paper is on people cooking together and particularly on how they arrange themselves spatially while cooking together. As other studies have done, our approach draws on data from YouTube (e.g. [2] [3] [12] [31]) using it both as a source for video and as "a community that serves as a platform for communication and interaction" [31].

\subsection{Using YouTube as Data Source}

An important task for HCI researchers interested in the design of new domestic technologies is to observe and make sense of people's daily practices, so that these can meaningfully inform design and seed innovation [1]. While this may sound simple, it actually presents a significant challenge within the context of people's homes, including their kitchens. As the movie "Kitchen Stories" [11] so charmingly illustrates, it is not always ideal, or even desirable, to do traditional direct observation in people's homes. This is also confirmed by research, like Crabtree and Rodden [5], concluding that although the home is relatively easy to access, such direct observation inevitably disrupts the ordinary flow of household activities and can cause people to alter their ordinary behavior.

In response to these challenges, research into the domestic domain has to a large extent turned to methods of self-reporting for obtaining insight into people's daily lives with minimal interference. This has been done through various techniques and data collection media, such as cultural probes (e.g. [6]), technology probes (e.g. [16]), and diaries (e.g. [27]). As an alternative approach, using YouTube as a data source represents a form of "digital ethnography" [22]. In digital ethnography, researchers immerse themselves in the digital world rather than physical and make use of digital data, such as written words, images, audio files, video and online communities, about the phenomenon of interest, which is often available in vast amounts today.

If chosen carefully, Blythe and Cairns argue that YouTube potentially provides several interesting avenues of inquiry as a digital on-line data source [2]. Using YouTube for studying cooking together potentially gives us insight on human behavior and activity in real world settings that are inherently difficult to study using traditional ethnographical technique. As people voluntarily and enthusiastically share a variety of 
different cooking situations with others on YouTube, we chose this as our data source, with "purposeful sampling" [30, p. 230], for a digital ethnography of how people cook together and share this activity digitally with others. In this way, we were able to rapidly gain access to a breadth of information about a variety of people in different generations, cultures and situations documenting their own experiences of "cooking together" (figure 2).

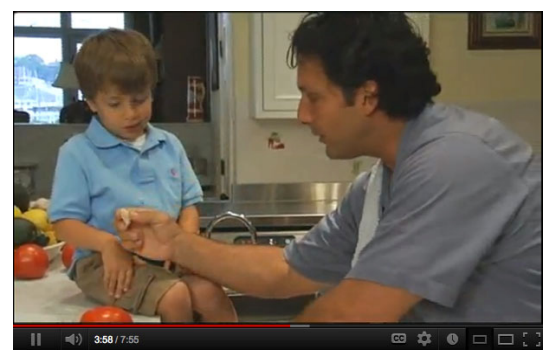

Fig. 2. YouTube video of "cooking together"

In studying the YouTube videos of people cooking together, our focus was on understanding the social aspects of cooking. In particular, we were interested in how people coordinate the activity, how they organize themselves spatially in respect to physical space and each other, the role of the food in this shared experience, and in particular how they include (or don't include) the viewer of the video in the interaction.

\subsection{Analysis}

Using the keyword phrase "cooking together", 169 videos were found on YouTube through a search on 15th November 2010. After discarding duplicates and unrelated videos (e.g. a song with the words "cooking together" in the lyrics), a final set of 61 videos of people cooking together remained. Inspired by Blythe and Cairns [3] [3], we conducted a qualitative content analysis of the 61 videos. This resulted in inductive development of the following categories: family life, family cooking, celebrities cooking, amateur cooking show, professional cooking show, documentary, educational and advertising.

Family life videos typically show people cooking together either in their own homes or outdoors with family or friends. The focus is on the people, their conversations and interactions - showing everyday life in the context of food preparation. There is little or no emphasis on what is being cooked. Family cooking videos have family members either taking on the roles of co-cooks, or parents and grandparents involving children in the cooking activity and teaching them how to participate. They are typically located in the family kitchen and sharing cooking techniques with each other is important but actual recipes are seldom elaborated to the viewer. Celebrities cooking differs from a cooking show, in that the celebrities are giving the viewer a "snapshot of their ordinary lives" in the context of cooking. These videos often include a background story about the celebrities public life interwoven 
with the activity of creating food. Amateur cooking show videos have the format of a professional television cooking show, with introduction, recipes, techniques and helpful hints, but are created by ordinary people from their home kitchens. They appear to be created specifically for YouTube where production company approval is not necessary to become the host of your own show. Professional cooking show videos are often versions of a show that runs on an established television or cable network. These shows have professional chefs and cooking celebrities, typically filmed in a television studio kitchen. Documentary videos are similar in some ways to the family life videos, in that there is story unfolding within the food context but in the documentry, people are sharing experiences or historical events with the viewing audience while cooking. Educational videos are usually made by a specific educational institution for disemination of cooking skills, to specific learning groups. Advertising videos, although often showing people cooking together, do so with the intention of attracting customers to cooking schools, public cooking events and hotels with special cooking weekends.

Secondly, the 61 videos were analyzed from the perspective of Kendon's Fformation system [18] [19]. In this analysis we looked for the following F-formations (for two people) L-shaped (standing orthogonal), vis-à-vis (facing) and side-by-side; and (for groups of three or more people) circular, rectangular, semi-circular and linear arrangements. We also identified spatial patterns of interaction between people, artifacts, spaces and the camera (viewer perspective) to give us insight into how people were sharing activities and interacting with others and artifacts in respect to the kitchen's physical layout. For this, we used Kendon's diagramming practice for recording F-formations [18] to "transcribe" the videos, recording patterns of behavior by creating a series of maps (figure 3) of the physical kitchen layout, showing the arrangements and movements of people and cameras within that space.

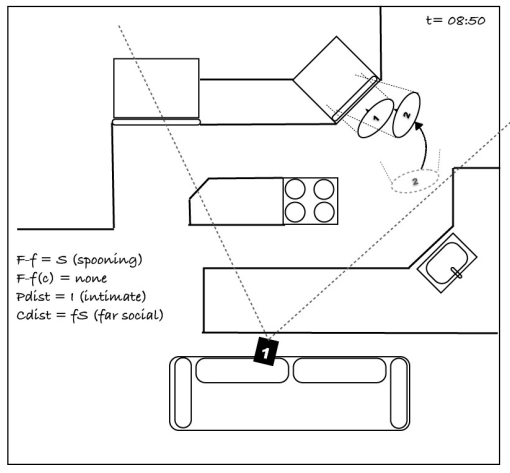

Fig. 3. F-formation Map of YouTube Video 57

Each map is time stamped $(t)$, representing elapsed video time, and records a newly established position of individuals. People are shown as numbered ovals with two lines extending to show their transactional segment, and hence where they intersect to create an F-formation. Previous positions (dotted) and paths of movement through spaces are represented, as are artifacts currently being used. We also documented the 
virtual position of the camera (black square) and the field of view (dotted lines) from that position. This made it possible to identify the viewer's participation in any Fformations. Each person's current activity, the focus of the current camera shot, and the general field of view as a description (e.g., half of kitchen bench top in view) were also noted. These maps were then analyzed to identify the following: the F-formation between co-located cooks $(F-f)$; the F-formation between us (the viewer) and the cooks $(F-f(c))$; the distance between cooks (Pdist); and the perceived distance between us (the viewer) and the cook (Cdist) using distance classifications of Hall [9]. For example, in figure $3, F-f=$ spooning, Pdist=intimate. This coding was then studied to identify spatial patterns supporting social connections.

\section{$4 \quad$ Findings}

In the following, we outline key findings from our analysis based on the spatial patterns unfolded in the videos. We identified patterns that aligned with the F-formations as described and illustrated by Kendon [19]. In addition to this, we also identified a number of new patterns not described in previous work.

In our analysis we identified a total of 1592 scenes in the 61 videos (a scene being defined as a move to a new person formation or a new camera position). No discernable formation was found in 663 scenes. The remaining 929 scenes involved two or more cooks in different formations and were analyzed and coded in detail.

\subsection{Known F-Formations}

In the analysis we identified patterns of interaction between cooks that aligned with Kendon's F-formations and were noticeably important for the shared cooking interaction. We identified the following four formations: Side-by-side, L-shaped, vis-à-vis, and semi-circular. Not surprisingly, we found no circular or rectangular formations as they would normally only occur for free-standing groups [21]. The following are examples of these four formations as they were instantiated in the activity of cooking together in their own kitchens, drawn from the sample YouTube videos.

The L-Shaped Formation. Kendon describes the L-shaped formation as participants standing orthogonal to each other [19]. This formation was also extensively used in the videos and was observed in 235 scenes across 43 videos. The L-shaped formation typically forms at the corner of a bench, table or stove-top, where cooks are positioned on two connected sides facing toward a central cooking task on which they are focused. Because this particular formation makes a very intimate shared central activity space, it often facilitates cooks working together on the same cooking task. One cook may be demonstrating a technique, either food preparation at a bench top or stirring food in a pot at a cook top, while the others watch.

Alternatively, it is a good configuration for passing a task backwards and forwards between participants. In the situation of working with small children, the child can 


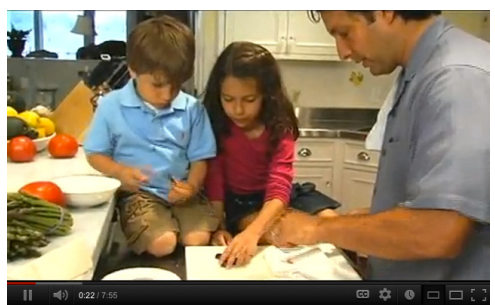

Fig. 4. A YouTube scene illustrating a L-shaped formation with three cooks

also be placed sitting on the bench top, so that they can see clearly and participate equally from that position in the food preparation (see figure 4).

The Side-by-Side Formation. The side-by-side formation occurred in 218 scenes. It was the single most used formation, and was observed in 46 videos. Side-by-side formation occurred in the kitchen when two or more cooks worked along one side of a shared bench or stove top. They may be working on a shared task, but more usually we observed that the cooks in side-by-side formation were working on concurrent but independent tasks (figure 5, bottom left). Another situation when this occurs is during instruction where one or more of the participants may be watching the main cook demonstrate a technique (figure 5, top left).
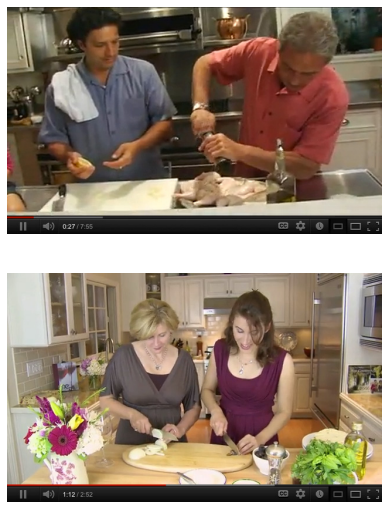

Side-by-side
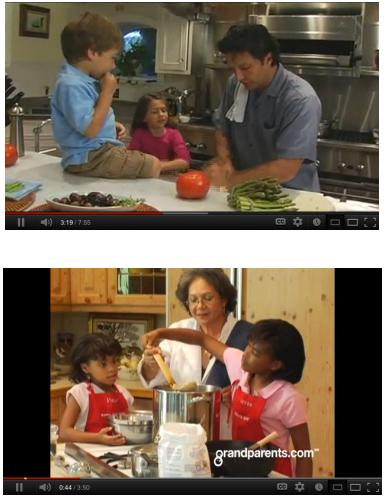

Semi-circular
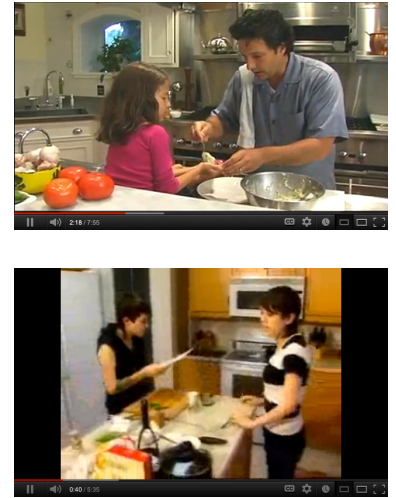

Vis-à-vis

Fig. 5. Different YouTube scenes illustrating three F-formations

With the added dimension of the camera in the interaction, the cooks may just be standing or sitting side be side and addressing the camera directly, hence facing the same direction while having a conversation and including the viewer. The use of sideby-side is not surprising as this formation illustrates people "that are jointly concerned about something in their immediate environment" [19]. In the kitchen, food acts as this "something" in the immediate environment and the kitchen layout (e.g. benches, table-tops) would sometimes support or enforce this formation. 
The Vis-à-Vis Formation. Vis-à-vis formations (where people arrange themselves face to face) were less common than the two previously illustrated formations. We identified vis-à-vis formations in 63 scenes from 25 videos. The layout of the kitchen certainly had an impact on how the vis-à-vis formation presented itself. The kitchen island design or a centrally placed kitchen table or cook-top with opposite sides accessible for working encourages this formation in food preparation. Cooks stand opposite each other while working on their cooking tasks and converse with each other face-to-face. In the scene in figure 5 (top middle), a father and his daughter work cooperatively on a shared task of shaping zucchini fritters. In the kitchen designs we observed, the width of the table or bench mostly precluded working on a shared task, but cooks could work cooperatively, in that one might be reading the recipe, while the other carries out the instructions (figure 5, bottom middle).

The Semi-Circular Formation. The semi-circular formation only happens in groups of more than two. In our sample videos, we identified 65 scenes from 16 videos involving three cooks. For these three cooks to form a semi-circle, they would generally be gathered around a shared task being carried out in a central shared space. The configuration is semi-circular in shape, that is, one side of the circle is open. This open side in the cooking together context was sometimes a consequence of the kitchen layout. This happened when people gathered around a stove or when younger children were placed sitting on the bench top arranged around the food preparation area (figure 5 , top right). In other instances, it appears that people in the kitchen have left one side of the circle open to give the camera a view of their activity (figure 5, bottom right).

\subsection{New Formations}

While we identified 581 spatial-orientational arrangements that could be described using the known formations from Kendon's work [18] [19], we also found 348 scenes that did not fit with this system. By analyzing these 348 scenes of different spatialorientational arrangements further, we have identified and named four additional types of formations according to their conversational characteristics. We have named these: wide V-shaped, Spooning, Z-shaped, and reverse L-shaped (figure 6).

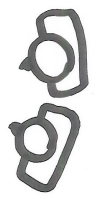

(a)

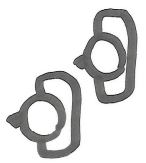

(b)

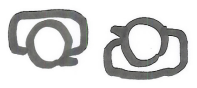

(c)

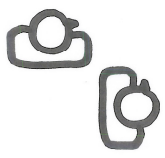

(d)

Fig. 6. Schematic examples of new formations:

(a) wide V-shaped, (b) Spooning, (c) Z-shaped, (d) reverse L-shaped

The following are examples of these four formations as they were instantiated in the activity of cooking together. 
The Wide V-Shaped Formation. The wide V-shaped formation is something that is peculiar to cooks positioned at a long bench top, where they are conversing or working closely together on a single task. We identified this formation in 163 scenes in 38 videos. In this formation, rather than having a side-by-side configuration, where they face forward, working in their own section of bench, they tilt their bodies slightly so that they are facing each other a bit more, but still actively engaging with an activity on the bench, or in the shared space between them. As an example, the two participants in figure 8 are having a conversation and the physical layout of the bench enforces this formation. Another example of a wide V-shaped formation was found when a father illustrates and instructs a child on how to cook.

We further noticed that if the wide V-shape becomes too acute then the participants lose connection with the bench and their cooking activity. There is a continuum of angles between the side-by-side and the L-shape formations but because of the way people work in kitchens and the popular and functional kitchen design of long bench spaces, cooking scenes often involved this very specific formation.

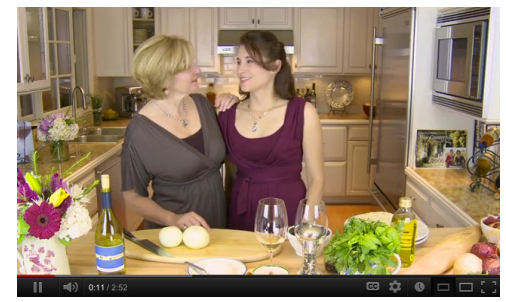

Fig. 7. A scene illustrating a wide V-shaped formation

The Spooning Formation. The spooning formation describes an intimate interaction important in showing and sharing progress while cooking together. We identified 80 spooning scenes in 31 of the 61 videos. When "spooning" in a kitchen, one cook would typically approach another cook from behind to assist and to see what they are doing. This could be in front of a stovetop, or kitchen bench. This means that they are facing the same direction and share the same immediate space in front of them both. From this position they can assist each other in a difficult task (figure 8, top left) or show details of their current activity, such as setting the oven temperature, to each other (figure 8 , bottom left). Sometimes this happens because there is only a small amount of space in which to show the second cook what the first is doing, such as when the first cook is working in a corner of the kitchen, or at a narrow sink.

The Z-Shaped Formation. The Z-shaped formation describes the interaction between two people cooking together while standing side-by-side but facing opposite directions. This formation was found in 57 scenes from 21 of the videos. Interaction takes place either by talking away from the other person, each person facing and talking forward, or by turning one's head towards the other while the torso and hands remain directed towards "ones' own work space". This is a configuration that would seldom happen in a conversational context, but is quite a comfortable way of interacting in the cooking together context. For example, a participant sitting on the bench 
top while talking to or interacting with the cook who is working at the same bench top (figure 8, bottom middle). Further, we saw this formation when cooks pass each other in the constrained environment of the kitchen, while keeping each other updated on current activity or simply continuing their conversation while moving between the variety of tasks and artifacts involved in preparing food (figure 8, top middle).
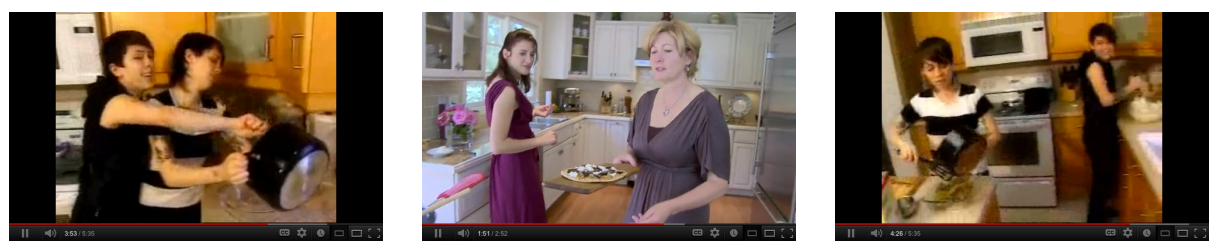

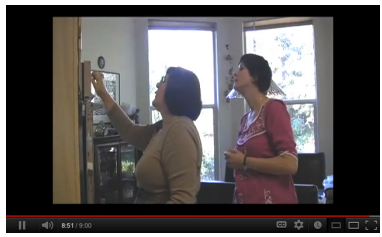

Spooning

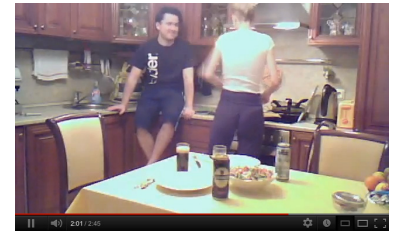

Z-shaped

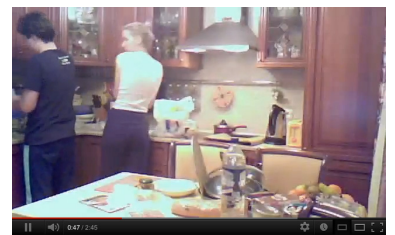

Reverse L-shaped

Fig. 8. Different YouTube scenes illustrating new formations

The Reverse L-Shaped Formation. The reverse L-shaped configuration is another configuration peculiar to the cooking environment. In this configuration, two cooks have their bodies in an L-shaped configuration, but they are facing away from each other. We identified this formation 48 times in 21 videos. In an L-shaped formation, cooks are evidently working on different tasks, but they are coordinating, cooperating and conversing as an integral part of the production of food. Kitchen layout plays a significant role in facilitating this formation, because it is sustained by working at a bench top, stove top or sink that places the two cooks in this position. Figure 8 (right) illustrates two different kitchens and two different examples of reverse L-shaped formations. In the bottom picture, the two cooks are standing in the corner of the kitchen, enforcing an L-shape, but in reverse. As it is quite usual in kitchen design to have benches along the walls of a square room, there are inevitably corners in which two bench-tops meet. Here, two cooks can be working at right angles to each other, while talking over their shoulders to each other, or leaning over to check each other's progress (figure 8, bottom right). Alternatively a central kitchen island makes it possible for one cook to work there, while the other works at a orthogonal bench behind them (figure 8, top right). Again the two cooks continue to converse and cooperate from this position periodically rotating their heads to visually engage with one another. 


\section{$5 \quad$ Discussion and Conclusions}

In this paper we have explored the experience of how people cook together with a particular emphasis on how they share these experiences through the use of a video camera. More specifically we were concerned with the ways that people oriented towards each other and a range of cooking artefacts in the context of these shared experiences and how such configurations were organized with respect to particular architectural features of the kitchen space. In adopting such analytic concerns, our aim was to provide a resource for thinking about how such activities might be enabled in distributed contexts. That is, by understanding some of the ways that people configure themselves both with respect to features of the local environment and other remote actors and audiences, how might we exploit these features in the configuration of distributed cooking experiences.

One of the ways that we have chosen to explore these concerns, and that forms the particular focus of the current paper, is through an analysis of a rather unusual data resource. This data resource consisted of corpus of existing YouTube videos created and posted by people documenting their various forms of shared cooking experiences. What is intriguing about such a resource is that it provides a view into a wide range of kitchen environments and cooking experiences, revealing both insights into local configuration patterns, but also some particular ways that people choose to organize and present themselves and their environment to a remote audience as mediated by a video camera. While the primary focus of the paper is with what is revealed about the shared cooking experience under such video mediated circumstances, a secondary concern is with some reflections on this unusual methodological approach.

\subsection{Spatial Arrangements in Cooking Together}

Drawing inspiration from Kendon's F-formation framework for understanding spatial configurations in social interactions, what was apparent from our video analysis was that there were a wide range of spatial configurations adopted by people in the context of sharing their cooking experiences. While some of these were of the vis-à-vis variety that one might typically associate with a standard videoconference model, what was of significance was the number of other configurations identified. Of these, we observed the use of three configurations that aligned closely with those identified in Kendon's original set of conversational arrangements, namely side-by-side, L-shaped, and semi-circular. But the analysis also revealed the use of four additional patterns at play in these contexts, namely wide $\mathrm{V}$-shape, spooning, Z-shape and reverse L-shape.

While the successful adoption of these varied different spatial configurations is of interest in itself, what is of particular concern is why such patterns are in evidence in these cooking experiences. That is, what is it about the activities that constitute these experiences and what is it about the environmental contexts that elicit these patterns? Firstly, kitchens are not free-form neutral spaces in which the collaborators can configure themselves without constraint. Rather, we see how people configure themselves with respect to particular physical features of the kitchen layout (e.g. benches, stoves, tables) as they engage in various cooking activities. As an example, several cooks 
from our videos formed L-shaped formations at the corner of a bench or stovetop where they would be positioned on two connected sides facing toward a central shared cooking task. It was the physical kitchen layout that encouraged the cooks to stand in the L-shaped pattern. Similarly, the side-by-side formations observed were generally due to a straight length of bench, which influenced their positioning, as they stood against it, next to each other. These physical constraints will shift and change in the ongoing trajectory of the cooking. Such constraints in the local environment, then, will be an important consideration for us in the context of potential distributed arrangements for these activities, in particular as they are accommodated over time.

Secondly, what we see in these new and varied forms of spatial configurations are a broader set of collaborative goals and activities and wider set of roles and ways in which people choose to participate. For example, people may work together on a task, they might perform separate activities in parallel, one might be showing others how to cook through visual demonstration or by guiding hands on-learning from behind. For some, the cooking is a focal point of the interaction, for others, it is an incidental part of being together - a context in which social interaction and conversation are managed even when attention is focused elsewhere. What we see in the analysis is how some of the particular spatial arrangements we observed were, in part, contingent on these activity characteristics wide, e.g. V-shaped formation (when two cooks are working at a long top bench) or spooning when teaching through hands-on learning.

The implications for design from these insights are subtle but important. Our study exemplified that cooking together is a somewhat complex activity and that installing a simple videoconference system in the kitchen will not cater for the rich social interactions taking place around food preparation that makes it a meaningful and positive experience. The identification of new formations when cooking together has inspired us to rethink the kinds of positioning, images and audio that need to be supported if we are to create a blended cooking experience for people distributed geographically.

In terms of camera and screen positions in kitchens, we are inspired to move away from the traditional fixed wide-angle and stovetop views towards more dynamic and intimate views supporting the shared cooking experiences that we observed. In particular, the Spooning formation draws us to consider technology configurations capable of reproducing close-up over-the-shoulder views to provide the intimate and informative aspect seen by the person behind. From the reverse L and Z-formations we noticed that in kitchens preparation and social interaction are interwoven, not discrete activities, and that although the cooking activity and kitchen architecture may draw cooks away from typical conversational formations, cooks continue talking to each other, even when facing opposing directions.

These observations indicate that any technology supporting the flow of activity and conversation could not do so from a single vantage point if it is to reproduce the intimacy of the person-to-person interaction. Audio sampling would need to take into account current body and head positioning of cooks, and a constant digital feed of images of the food, the cooks or both may not even be necessary. At the same time, we can see that moving about, working on different cooking tasks and swiveling your head to engage with the other person works well in particular patterns within the kitchen space. The implication being that given a particular spatial kitchen design it 
should be possible to predict locations for screens to provide an effective blended cooking experience. These are design implications that we will explore in the future, and that we encourage others to consider too.

\subsection{Digital Ethnography for Design}

Methodologically we have explored a "digital ethnography" approach to gaining insight into activities in everyday life in people's private homes through user-created videos posted on YouTube. While this is not the first study in HCI research to do so, we still think it is worth reflecting a bit on this approach to empirical studies in domestic settings for the purpose of informing interaction design.

Two things one could criticize the YouTube approach to ethnography for are that 1) it might not provide a representative picture of the behavior of the general population, and 2) the presence of the camera may cause people to behave differently than they normally would. These are valid concerns and certainly warrant further thinking. They are, however, not new concerns pertaining only to the use of YouTube videos, but known issues in empirical research for domestic computing. Our perspective on the subject is that in order to make reasonable judgment about methodological appropriateness one needs to consider the purpose of the study. From this perspective, many empirical user studies in HCI research differ considerably from empirical studies of people in, for example, sociology and anthropology, where ethnography has its origins. In contrast, HCI researchers might not be aiming at theory about people and society as such, but instead "just" be interested in informing and inspiring interesting and novel interaction design. In this case, traditional ethnography might be a hindrance rather than an enabler of innovation. To deliberately focus on "extreme" users rather than mainstream (the IDEO approach) can sometimes be more useful for design. In our experience from this study, we found that using YouTube as our data source enabled us to gain understanding and inspiration valuable for future design. We were able to observe a broad cohort of people doing interesting social interaction in their kitchens beyond what we possibly could have achieved in a similar time frame by going into the field. Furthermore we were able to do purposeful sampling [30] and focus on particular observations and emergent themes as the study evolved.

In terms of the camera influencing people's behavior it was our clear impression that this was not a threat to validity. On the contrary, the obvious presence of the camera sometimes actually added to the insight gained, as people would be using it actively in communicating with the remote viewers - something in itself of great importance when interested in supporting distributed social cooking. One could say that the presence of cameras in people's kitchens was so overt that it was almost covert.

\subsection{Designing Technologies for Cooking Together}

The implications for design from these insights are subtle but important. Our study exemplified that cooking together is a somewhat complex activity and that installing a simple videoconference system in the kitchen will not cater for the rich social interactions taking place around food preparation that makes it a meaningful and positive experience. The identification of new formations when cooking together has inspired 
us to rethink the kinds of positioning, images and audio that need to be supported if we are to create a blended cooking experience for people distributed geographically. In terms of camera and screen positions in kitchens, we are inspired to move away from the traditional fixed wide-angle and stovetop views towards more dynamic and intimate views. In particular, the Spooning formation draws us to consider technology configurations capable of reproducing close-up over-the-shoulder views to provide the aspect seen by the person behind. From the reverse $\mathrm{L}$ and $\mathrm{Z}$-formations we noticed that cooking and conversations are interwoven, not discrete activities, and that although the cooking activity and kitchen architecture may draw cooks away from typical conversational formations, they continue talking to each other, even when facing opposing directions. These observations indicate that any technology supporting the flow of activity and conversation could not do so from a single vantage point if it is to reproduce the intimacy of the person-to-person interaction. Audio sampling would need to take into account current body and head positioning of cooks, and a constant digital feed of images of the food, the cooks or both may not even be necessary. At the same time, we can see that moving about, working on different cooking tasks and swiveling your head to engage with the other person works well in particular patterns within the kitchen space. The implication being that given a particular spatial kitchen design it should be possible to predict locations for screens to provide an effective blended cooking experience. These are design implications that we will explore in the future, and that we encourage others to consider too.

Acknowledgements. We would like to thank Adam Kendon for valuable comments on our research.

\section{References}

1. Bell, G., Kaye, J.: Designing technology for domestic spaces: A Kitchen Manifesto. Gastronomica, 46-62 (2002)

2. Blythe, M., Cairns, P.: Critical Methods and User Generated Content: the iPhone on YouTube. In: Proc. CHI 2009, pp. 1467-1476. ACM (2009)

3. Blythe, M., Cairns, P.: Tenori-On Stage: YouTube As Performance Space. In: Proc. NordiCHI 2010, pp. 72-81. ACM (2010)

4. Bonanni, L., Lee, C.-H., Selker, T.: Cooking with the Elements: Intuitive Immersive Interfaces for Augmented Reality Environments. In: Costabile, M.F., Paternó, F. (eds.) INTERACT 2005. LNCS, vol. 3585, pp. 1022-1025. Springer, Heidelberg (2005)

5. Crabtree, A., Rodden, T.: Domestic Routines and Design for the Home. Computer Supported Cooperative Work 13(2), 191-220 (2004)

6. Gaver, B., Dunne, T., Pacenti, E.: Design: Cultural probes. Interactions 6(1), 21-29 (1999)

7. Greenberg, S., Marquardt, N., Ballendat, T., Diaz-Marino, R., Wang, M.: Proxemic interactions: the new ubicomp? Interactions 18(1), 42-50 (2011)

8. Grimes, A., Harper, R.: Celebratory Technology: New Directions for Food Research in HCI. In: Proc. CHI 2008, pp. 467-476. ACM (2008)

9. Hall, E.T.: The Hidden Dimension. Anchor Books (1966)

10. Hamada, R., Okabe, J., Ide, I., Satoh, S., Sakai, S., Tanaka, H.: Cooking Navi: Assistant for Daily Cooking. In: Proc. Multimedia 2005, pp. 371-374. ACM (2005)

11. Hamer, B.: Kitchen Stories (Salmer fra kjøkkenet) [Motion picture]. Norway (2003) 
12. Harley, S., Fitzpatrick, G.: Creating a conversational context through video blogging. Computers in Human Behaviour 25(3), 679-689 (2009)

13. Heaton, L.: Preserving Communication Context: Virtual Workspace and Interpersonal Space in Japanese CSCW. In: Proc. Cultural Attitudes Towards Communication and Technology 1998, pp. 207-230. University of Sydney, Australia (1998)

14. Hung, H., Kröse, B.: Detecting F-formations as Dominant Sets. In: Proc. ICMI 2011, pp. 231-238 (2011)

15. Huettenrauch, H., Eklundh, K., Green, A., Topp, A.: Investigating Spatial Relationships in Human-Robot Interaction. In: Proc. IROS 2006, pp. 5052-5059 (2006)

16. Hutchinson, H., Mackay, W., Westerlund, B., et al.: Technology probes: inspiring design for and with families. In: Proc. CHI 2003, pp. 17-24. ACM (2003)

17. Ju, W., Hurwitz, R., Judd, T., Lee, B.: CounterActive: An Interactive Cookbook for the Kitchen Counter. Ext. Abstracts CHI 2001, pp. 269-270. ACM (2001)

18. Kendon, A.: Conducting Interaction: Patterns of Behavior in Focused Encounters. Cambridge University Press, Cambridge (1990)

19. Kendon, A.: Spacing and orientation in co-present interaction. In: Esposito, A., Campbell, N., Vogel, C., Hussain, A., Nijholt, A. (eds.) COST 2102 Int. Training School 2009 LNCS, vol. 5967, pp. 1-15. Springer, Heidelberg (2010)

20. Lagomarsino, A.: Kitchen album: concept based on progressive user research. In: Proc. DPPI 2007, pp. 423-428. ACM (2007)

21. Marshall, P., Rogers, Y., Pantidi, N.: Using F-formations to analyse spatial patterns of interaction in physical environments. In: Proc. CSCW 2011, pp. 445-454. ACM (2011)

22. Masten, D.L., Plowman, T.M.P.: Digital Ethnography: The next wave in understanding consumer experience. Design Management Journal 14(2), 75-81 (2003)

23. Mennicken, S., Karrer, T., Russell, P., Borchers, J.: First-Person Cooking: A DualPerspective Interactive Kitchen Counter. Ext. Ab. CHI 2010, pp. 3403-3408. ACM (2010)

24. Mentis, H., O'Hara, K., Sellen, A., Trivedi, R.: Interaction proxemics and image use in neurosurgery. In: Proc. CHI 2012, pp. 927-936. ACM (2012)

25. Morrison, C., Jones, M., Blackwell, A., Vuylsteke, A.: Electronic patient record use during ward rounds: a qualitative study of interaction between medical staff. Critical Care 12(6), R148 (2008)

26. Nardi, B., Schiano, D., Gumbrecht, M., Swartz, L.: Why we blog. Communications of the ACM - The Blogsphere 47(12), 41-46 (2004)

27. Palen, L., Hughes, A.: When home base is not a place: parents' use of mobile telephones. Pers. Ubiquit. Computing 11(5), 339-348 (2007)

28. Obata, A., Sasaki, K.: Office Walker: a virtual visiting system based on proxemics. In: Proc. CSCW 1998, pp. 1-10. ACM (1998)

29. O'Hara, K., Kjeldskov, J., Paay, J.: Blended interaction spaces for distributed team collaboration. ACM TOCHI 18(1), article no. 3 (2011)

30. Patton, M.: Qualitative Research \& Evaluation Methods. Sage Publications, Thousand Oaks (2002)

31. Rotman, D., Preece, J.: The 'WeTube' in YouTube - creating an online community through video sharing. Int. J. Web Based Communities 6(3), 317-333 (2010)

32. Rogers, Y.: HCI Theory: Classical, Modern, and Contemporary. Morgan \& Claypool (2012)

33. de Runa, J., Harpring, J., Rafiuddin, M., Zhu, M.: Not Enough Cooks in the Kitchen. In: Proc. CSCW 2010, pp. 485-486. ACM (2010)

34. Terrenghi, L., Hilliges, O., Butz, A.: Kitchen Stories: sharing recipes with the Living Cookbook. Pers. Ubiquit. Computing 11(5), 409-414 (2007)

35. Tran, Q.T., Calcaterra, G., Mynatt, E.D.: Cook's Collage: Deja Vu Display for a Home Kitchen. In: Sloane, A. (ed.) HOIT 2005. IFIP, vol. 178, pp. 15-32. Springer, Boston (2005) 